\title{
Predictive study of high performance scenarios in HL-2A
}

\author{
Q.D. Gao ${ }^{1}$, R.V. Budny ${ }^{2}$, F. Li ${ }^{1}$ and J. Zhang ${ }^{1}$ \\ ${ }^{1}$ Southwestern Institute of Physics, PO Box 432, Chengdu 610041, \\ Peoples's Republic of China \\ 2 Princeton Plasma Physics Laboratory, Princeton University, Princeton, NJ 08543, USA \\ E-mail: qgao@swip.ac.cn
}

Received 7 November 2002, accepted for publication 7 July 2003

Published 28 August 2003

Online at stacks.iop.org/NF/43/982

\begin{abstract}
High performance scenarios in the HL-2A tokamak are studied by numerical modelling. Through shifting the plasma column outwards, a shaped plasma with significant triangularity is achieved with sufficient room left for the RF antenna. For the out-shifted, shaped plasma, ripple loss of high energy ions during neutral beam injection (NBI) is analysed, and the results show that the ripple loss fraction of NBI power for the shaped plasma is no higher than that for the unshifted circular plasma. The time dependent TRANSP code is used to model realistic reversed magnetic shear (RS) operation in such plasmas. In order to sustain the RS operation towards steady-state, an off-axis current drive with a lower hybrid wave at $2.45 \mathrm{GHz}$ is used to control the current profile. A steady-state RS discharge is formed and sustained until the LH power is turned off; the plasma confinement is enhanced with the development of an internal transport barrier. In the RS discharges with shaped plasma geometry, a double transport barrier is developed. To understand the underlying physics for the current profile control with LHCD in HL-2A, the LH wave deposition in plasmas with RS is analysed.
\end{abstract}

PACS numbers: 52.55.Fa, 52.55.Wq, 52.25.Fi

\section{Introduction}

The construction of HL-2A [1], the first divertor tokamak in China, was accomplished recently at SWIP, Chengdu, and it will be ready for regular operation soon. The major objectives of HL-2A are to produce more adaptable divertor configurations to study energy exhaust and impurity control, and to enhance plasma confinement by profile control. A high density plasma, which is required for the cold and dense divertor, will degrade the plasma confinement. Recent experiments on JET, DIII-D, JT-60U and ASDEX Upgrade [2-5] have shown positive effects from increased triangularity on confinement. By using the flexible power supply system of the poloidal field coils in HL-2A, shaping plasmas with significant triangularity can be produced.

The studies $[6,7]$ on the optimization of the current density profile suggest that reversed magnetic shear (RS) is desirable for confinement, stability and bootstrap alignment. In many tokamaks, RS plasmas develop an internal transport barrier (ITB) that produces improved central confinement. However, the RS discharges established in the early experiments were usually transient in nature due to the development of MHD instabilities. The experimental observation of greatly reduced transport in RS plasma provided a strong motivation to further explore current profile control by which optimized RS operation may be established and maintained for timescales beyond the characteristic current diffusion time. In HL-2A, the various schemes of auxiliary heating and current drive, including neutral beam injection (NBI) (3 MW), ICRH (1 MW), LHCD (2.0 MW) and ECRH (0.5 MW), offer opportunities to optimize the current profile. In Tore Supra discharges [8], stable and stationary RS plasmas have been achieved with LHCD during reduced magnetic operation, and these plasmas are of a rather low $\beta$ value $\left(\beta_{\mathrm{N}} \approx 0.9\right)$. We employ LHCD to control the current profile in the HL-2A plasma heated by higher power NBI $\left(P_{\mathrm{NB}} \sim 2 \mathrm{MW}\right)$ to establish quasi-stationary RS configurations in higher $\beta$ plasmas.

For a tokamak device to have enhanced confinement, it is usually expected that it operates in the high, or H-mode. An edge transport barrier, or a region of reduced energy transport, characterizes this regime. In the tokamak experiments [9-11], an ITB can be developed and maintained inside the $\mathrm{H}$-mode edge barrier, producing an operating regime called the double transport barrier (DB) regime, and this operating regime improves the plasma performance substantially. In the HL-2A divertor plasma with strong shaping, an RS discharge with DB is also a candidate mode for high performance operation.

The load assembly of the former ASDEX tokamak is used in HL-2A. The shifting of the plasma column out to a higher major radius is one of the key operational changes 
that produces stronger shaping and therefore permits high performance scenarios. In this paper, we model quasistationary high performance modes of operation in HL-2A established with plasma shaping and profile control. In the next section, the shaped plasma geometries are computed. As the plasma shaping is achieved by moving the plasma column outwards where the toroidal magnetic field (TF) ripple increases rapidly, and as the TF ripple loss of NBI particles is important in the device operation, we analyse the ripple loss by using the Goldston-White-Boozer model [12]. In section 3, we first study the high performance scenarios for the circular plasma. Using current profile control to form an RS configuration, a quasi-stationary ITB mode with L-mode edge is established, and the mode is in the enhanced confinement regime and maintained by nearly full non-inductive current. Then, we discuss the high performance scenarios for the shaped plasma. As the high pressure gradient produced by $\mathrm{H}$-mode at the plasma edge can be allowed through plasma shaping, in the shaped plasma a DB confinement mode with H-mode edge is obtained by profile control. Comparing with the ITB case in the circular plasma geometry, higher plasma parameters are obtained in the DB regime. Finally, we analyse the LH wave deposition in plasmas with RS. In high temperature reactor grade plasmas, LH waves can be deposited in the outer region by strong Landau damping. Nevertheless, the plasma temperature in HL-2A is much lower than that in a future reactor, and the plasma absorption of high phase velocity $\mathrm{LH}$ waves is too weak to ensure the waves are damped directly in the outer region. Therefore, to achieve off-axis LH power deposition in the weak damping regime, we rely upon the wave propagation conditions constrained by the LH wave dispersion relation in a magnetized plasma. It now appears that by choosing the plasma parameters properly, the wave propagation domain in the toroidal plasma column is limited to the outer region, which naturally prevents any central LH power deposition irrespective of the toroidal broadening of the initially launched spectrum.

\section{Plasma shaping}

Although the divertor coils are fixed in a vacuum vessel in HL-2A, plasma shaping is possible through shifting the plasma column outwards. By reasonable adjustment of the currents in the vertical field coils and compensatory coils, shaped plasmas with significant triangularity have been achieved. The plasma equilibrium geometry is calculated with a freeboundary equilibrium code (SWEQU), in which the plasma current is modelled by

$j_{\mathrm{p}}=j_{0}\left[\beta_{\mathrm{p} 0} \frac{R}{R_{0}}+\left(1+\beta_{\mathrm{p} 0}\right) \frac{R_{0}}{R}\right]\left(\frac{\psi-\psi_{\mathrm{b}}}{\psi_{0}-\psi_{\mathrm{b}}}\right)^{\alpha_{1}}\left(\alpha_{2}+\rho\right)$

where $\rho=\left(\psi_{0}-\psi\right) /\left(\psi_{0}-\psi_{\mathrm{b}}\right)$ with $\psi_{\mathrm{b}}$ and $\psi_{0}$ being the poloidal flux at the boundary and the magnetic axis, respectively, $j_{\mathrm{p}}$ is the plasma current density, $j_{0}$ a constant defined by the total plasma current, $R_{0}$ the major radius of the plasma centre, $\beta_{\mathrm{p} 0}$ a poloidal beta related constant, and $\alpha_{1}$, $\alpha_{2}$ the current profile constants. Two typical shaped plasma geometries were produced: a D-shape plasma (figure 1 $(a)$ ) and an elongated D-shape plasma (figure $1(b)$ ). The main
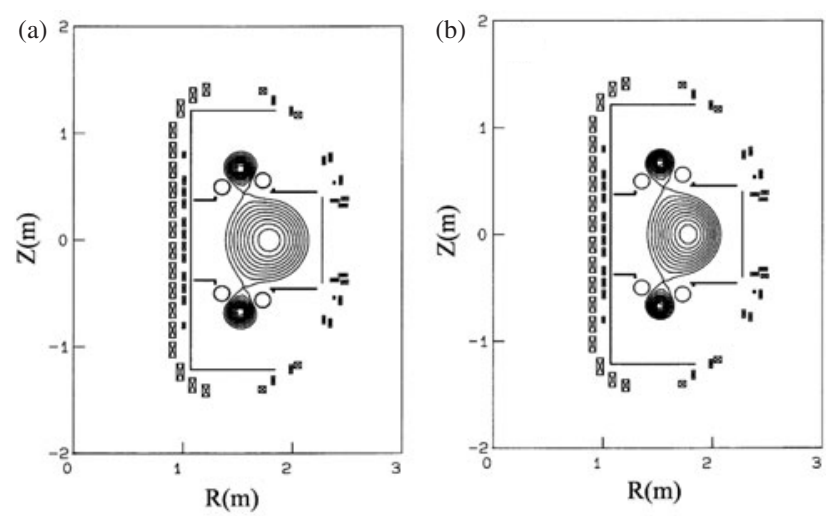

Figure 1. Magnetic geometries of the out-shifted plasmas: (a) D-shaped plasma, (b) elongated D-shaped plasma.

equilibrium parameters are given in table 1 , where the plasma parameters are obtained from the simulation of the stationary RS discharge by using the corresponding shaped geometry.

For the shaped plasma geometries, the triangularity variation with respect to the flux coordinate is dependent on the plasma current profile, but for both hollow and peaked current profiles it decreases rapidly at the plasma boundary region while moving towards the plasma centre (figure 2 ). The $\mathrm{H}$-mode transport barrier is localized at the plasma edge, and the pressure of the H-mode pedestal increases strongly with triangularity due to the increase in the margin by which the edge pressure gradient exceeds the ideal ballooning mode limit [3]; therefore, the rather high triangularity located at the plasma edge is favourable to enhance the confinement.

For the out-shifted, shaped plasma, the TF ripple loss of high energy ions during neutral beam injection (NBI) is estimated by assuming the trapped ions lost if their turning points are in regions where the ripple exceeds an empirical factor times the Goldston-White-Boozer threshold [12]. Ionization and capture of NBI particles, and slowing down of high energy ions, are calculated with a Monte Carlo technique. The ripple loss, orbit loss, and charge-exchange loss are calculated separately. The TF ripple $\varepsilon(R, Z) \equiv$ $\left(B_{\max }-B_{\min }\right) /\left(B_{\max }+B_{\min }\right)$ generated by $16 \mathrm{D}$-shaped coils is calculated, showing that its value at the outer edge of the $\mathrm{D}$-shaped plasma is rather high (close to $2 \%$ ). The toroidal ripple contours are of an elongated D-shape, as shown in figure 3, where the shift of the plasma position is also shown. In order to show whether the ripple loss for the out-shifted plasma can be tolerated we made a comparison for the ripple loss between a shaped plasma ( $D$-shaped plasma as shown in figure $1(a)$ ) and the unshifted circular plasma. The results show that the ripple loss fraction of NBI power for the shaped plasma is no higher than that for the circular plasma (figure 4) because most high energy ions are not deeply trapped in the case of tangential NBI.

\section{RS operation}

\subsection{Quasi-stationary RS discharges with ITB}

The time dependent TRANSP code [13] is used to model realistic RS operation in HL-2A. In order to sustain the RS 
Table 1. Main equilibrium parameters of the two shaped plasmas ${ }^{1}$

\begin{tabular}{llllllllllll}
\hline & $R_{0}(\mathrm{~m})$ & $a(\mathrm{~m})$ & $B_{\mathrm{T}}(\mathrm{T})$ & $I_{\mathrm{p}}(\mathrm{kA})$ & $k_{95}$ & $\delta_{95}$ & $\bar{n}_{\mathrm{e}}\left(m^{-3}\right)$ & $n_{\mathrm{e} 0} /\left\langle n_{\mathrm{e}}\right\rangle$ & $\beta_{\mathrm{N}}$ & $l_{\mathrm{i}}$ & $p_{0} /\langle p\rangle$ \\
\hline Figure 1 $(a)$ & 1.76 & 0.38 & 2.8 & 265 & 1.08 & 0.44 & $2.50 \times 10^{19}$ & 1.86 & 1.51 & 1.03 & 3.4 \\
Figure 1 $(b)$ & 1.74 & 0.33 & 2.8 & 265 & 1.21 & 0.41 & $2.39 \times 10^{19}$ & 1.88 & 1.58 & 1.01 & 3.5 \\
\hline
\end{tabular}

${ }^{1} k_{95}$ and $\delta_{95}$ are the elongation and triangularity of the $95 \%$ flux surface, respectively; $n_{\mathrm{e} 0} /\left\langle n_{\mathrm{e}}\right\rangle$ and $p_{0} /\langle p\rangle$ indicate the profile peaking of the plasma density and pressure, respectively (where \langle\rangle denotes volume averaging).

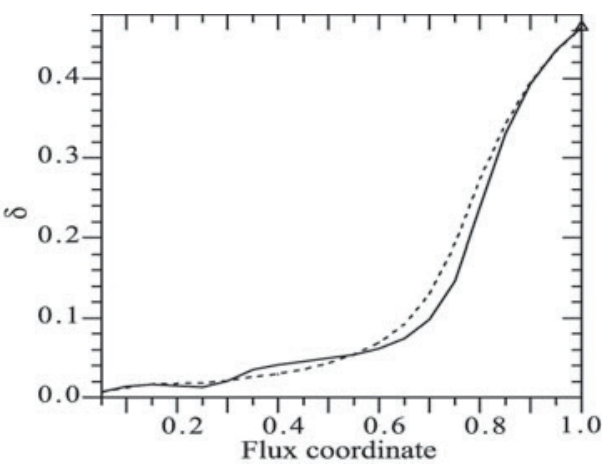

Figure 2. Triangularity of the $\mathrm{D}$-shaped plasma, $\delta$ vs the flux surface for the cases of hollow current profile $(-)$ and peaked current profile $(\cdots \cdots)$.

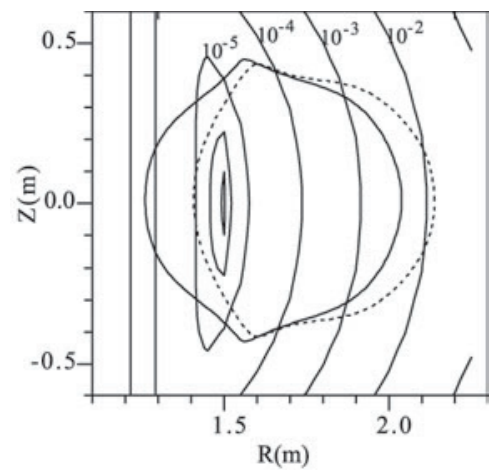

Figure 3. Contours of the toroidal field ripple. The locations of the circular plasma boundary ( - ) and D-shaped plasma boundary $(\ldots \ldots)$ are also shown.

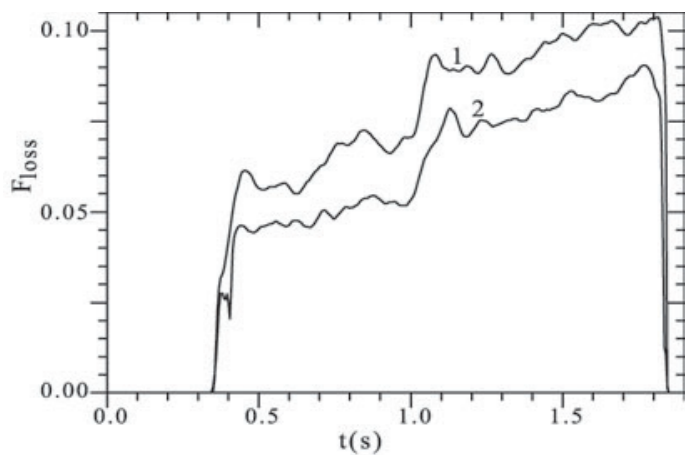

Figure 4. Ripple loss fraction of the NBI power, $F_{\text {loss }}$ vs time for a circular plasma (curve 1) and an out-shifted (D-shaped) plasma (curve 2).

operation towards steady-state, off-axis current drive with lower hybrid (LHCD) waves at $2.45 \mathrm{GHz}$ is used to control the current profile. The target plasma is a circular plasma of $I_{\mathrm{p}}=265 \mathrm{kA}$ with a modestly peaked density profile $\left(n_{\mathrm{e}}(0) /\left\langle n_{\mathrm{e}}\right\rangle=1.86, \bar{n}_{\mathrm{e}}=2.31 \times 10^{19} \mathrm{~m}^{-3}\right)$, which is heated by means of a $2.0 \mathrm{MW}$ neutral beam (1.5 MW co-injection and $0.5 \mathrm{MW}$ counter-injection).

The LH wave power spectrum radiated by a multi-junction launcher $(2 \times 12)$ is calculated using Brambilla coupling theory [14]. The LHCD calculation [15] utilizes a toroidal ray-tracing for the wave propagation and a parallel velocity Fokker-Planck calculation for the interaction of waves and particles. The LHCD package combined with the Brambilla coupling calculation is used in conjunction with the TRANSP code to obtain the driven current in a dynamic case.

The energy transport model is a mixed theory model [1]. Normally, the transport observed in tokamak experiments greatly exceeds that of collisional transport theory and this anomalous transport is usually attributed to turbulent fluctuation arising from various micro-instabilities. However, no theoretical model has yet been proposed that adequately describes all of the many features displayed by tokamak transport. Since ion energy transport drops to roughly the neoclassical level in RS discharges, the ion heat diffusivity is assumed in terms of neoclassical transport enhanced by $\eta_{\mathrm{i}}$ turbulence, which is responsible for the transport outside the shear reversal region. The electron energy transport is based on the Rebut-Lallia-Watkins (RLW) model [16], which, from heuristic and dimensional arguments, introduces a critical electron temperature gradient $\nabla T_{\mathrm{ec}}$ such that the electron heat flow is neoclassical when $\left|\nabla T_{\mathrm{e}}\right|<\left|\nabla T_{\mathrm{ec}}\right|$ or $\nabla q<0$. The distinctive feature of this hybrid model is that both the $\eta_{\mathrm{i}}$ model and the RLW model have been tested against a wide range tokamak devices [17]. The ion temperature gradient (ITG), or $\eta_{\mathrm{i}}$ driven fluctuations, are drift waves with $k_{\perp} \leqslant \rho_{\mathrm{i}}$, where $k_{\perp}$ is the perpendicular wavenumber of the fluctuation and $\rho_{\mathrm{i}}$ the ion gyro-radius. The RLW model is a gyro-Bohm model that would be appropriate for diffusive transport due to fluctuations on a micro-scale $\rho_{\mathrm{i}}$. Thus, it is expected that the transport model used in the simulation gives a better description for the transport produced by short wavelength turbulence, but probably the contribution resulting from the toroidal coupling of long wavelength turbulence is not adequately treated.

Due to the off-axis LHCD combined with the effect of bootstrap current and beam driven current, a steady-state RS discharge is formed and sustained with the minimum $q$ value, $q_{\min } \approx 2.8$ and the location of $q_{\min }, x_{\min } \approx 0.65$ until the LH power is turned off (figure 5). In earlier experiments, the RS plasmas were usually created by heating the plasma during the current ramping-up phase and maintained by rather high fraction of off-axis bootstrap current, which would cause highly peaked pressure profiles (e.g. $p_{0} /\langle p\rangle$ is higher than 5.0 for the RS plasmas in TFTR and the NCS plasmas in DIII-D); however, excessive peaking of the pressure can lead to instability of low- $n$ modes, for example, the disruption of 
RS discharges in TFTR is identified to be caused by an ideal $n=1$ instability, an infernal/kink mode [18], and the bursting MHD activity observed near the disruption boundary of NCS (negative central shear) discharges in DIII-D is the resistive interchange modes [19]. The theoretical investigations on the MHD operational limits of the RS tokamak [20-22] also conclude that the optimal pressure profiles are never strongly peaking. The HL-2A RS discharge produced with the NBI heating started during the current ramping-up phase has also been modelled and analysed with respect to several MHD instability modes [23]. The RS equilibria with highly peaked pressure profile are not stable against several MHD modes, especially the low- $n$ internal modes located in the low shear region are unstable. To avoid excessively peaked pressure profile, the pressure profile has been controlled by stepdown of beam injection power in JT-60U, and a quasi-steady sustainment of RS discharges was achieved [24]. Here, LHCD is employed to control the current profile separately while keeping the pressure profile modestly peaked $\left(p_{0} /\langle p\rangle \approx 3.5\right)$ so as to prevent pressure driven internal mode instabilities.

In the quasi-stationary RS discharge, plasma confinement is enhanced with the development of an ITB. The ITB manifests itself by the higher gradient of ion temperature inside it. It is maintained in a stationary state during the whole RS phase, and the position of the maximum $\left|\nabla T_{\mathrm{i}}\right|$, where the minimum of ion heat diffusivity is located, is near $x_{\min }$. When the reversed shear exists, the location of the ITB temporally evolves following the evolution of the shear reversal point (figure 5(c)). The enhancement factor over ELMy H-mode as given by the $\operatorname{IPB} 98(y, 2)$ scaling [25] is $H_{98(y, 2)} \approx 1.1$, and the normalized $\beta$ value is $\beta_{\mathrm{N}} \approx 1.4$ (figure $5(b)$ ). This RS discharge has nearly achieved a fully non-inductive current

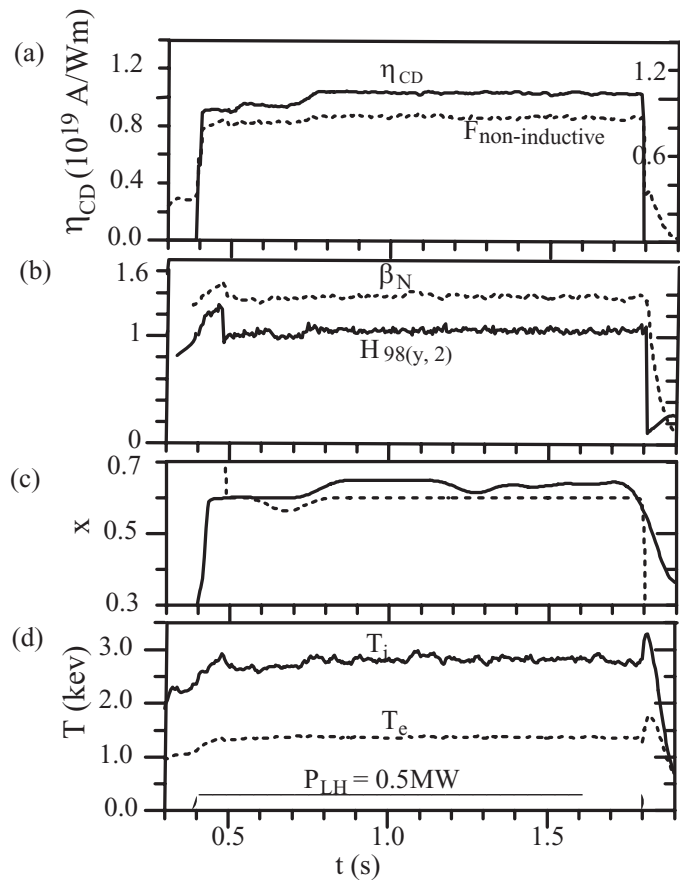

Figure 5. Time traces of a quasi-stationary RS discharge: (a) LHCD efficiency, $\eta_{\mathrm{CD}}$ and non-inductive current fraction, $F_{\text {non-inductive }}$; (b) the H-factor, $H_{98(y, 2)}$ and normalized beta, $\beta_{\mathrm{N}} ;(c)$ the locations of the minimum $q\left(\stackrel{)}{\longrightarrow}\right.$ and the minimum $\chi_{\mathrm{i}}(\cdots \cdots) ;(d)$ the central plasma temperatures $\left(T_{\mathrm{i}}, T_{\mathrm{e}}\right)$. drive with a non-inductive current (which includes LH wave driven current, NBI driven current and bootstrap current) fraction of $\sim 90 \%$ of the total plasma current and an LHCD efficiency $\eta_{\mathrm{CD}} \approx 1.0 \times 10^{19} \mathrm{~A} \mathrm{Wm}^{-2}$ (figure $5(a)$ ).

The sustainable RS scenario is robust. We have performed the RS discharge modelling for various different target plasmas, such as that for both fully co-injected neutral beam and approximately balanced beam injections (1.0 MW co-injection and $0.8 \mathrm{MW}$ counter-injection), producing beam driven currents of $I_{\mathrm{NB}} \sim 100 \mathrm{kA}$ and $I_{\mathrm{NB}} \sim 10 \mathrm{kA}$, respectively (compared to $I_{\mathrm{NB}} \sim 45 \mathrm{kA}$ in the standard target plasma); the NBI heating power decreases by $\sim 25 \%$, and the plasma temperature is accordingly reduced to $T_{\mathrm{e} 0} \approx 1.0 \mathrm{keV}$, $T_{\mathrm{i} 0} \approx 2.2 \mathrm{keV}$ from $T_{\mathrm{e} 0} \approx 1.4 \mathrm{keV}, T_{\mathrm{i} 0} \approx 2.8 \mathrm{keV}$ in the standard case; the peaking factor of the density profile $n_{\mathrm{e}}(0) /\left\langle n_{\mathrm{e}}\right\rangle$ increases by $\sim 25 \%$; and the total plasma density increases by $25 \%$. In all these cases, similar off-axis noninductive driven current profiles are produced during the steady-state phase, as in the standard case, and sustained RS discharges are achieved.

\section{2. $R S$ discharge with $D B$}

The elongated D-shaped plasma shown in figure 1(b) $\left(\delta_{98}=0.43, k_{98}=1.23\right)$ is used to model the RS discharge. The geometry of the boundary ( $98 \%$ flux surface of the diverted plasma) is specified as a general function of time, which evolves from circular to elongated D-shape during the current ramping-up phase and then retains the same shaped boundary in the current flat-top phase. The interior flux surfaces, which are computed by solving the Grad-Shafranov equation, are parameterized by the square-root of the normalized toroidal flux, $\rho$.

The standard target plasma described above is used, but the electron density profile has a modest change with a more obvious edge pedestal. The current profile is still controlled by LHCD. With the lower hybrid power $\left(P_{\mathrm{LH}}=0.5 \mathrm{MW}\right)$ switched on, a steady-state RS discharge is produced with a DB developed for the shaped plasma. The DB is indicated by two abrupt decreases of the ion heat diffusivity (figure 6), of which the two minima are located near the shear reversal point, $\rho_{\min } \approx 0.55$, and near the plasma edge, $\rho \approx 0.95$. The elevated heat diffusivity between the two minima separates the two barriers. The transport barriers are also shown on the ion temperature profile (figure 7), where the shaded regions are

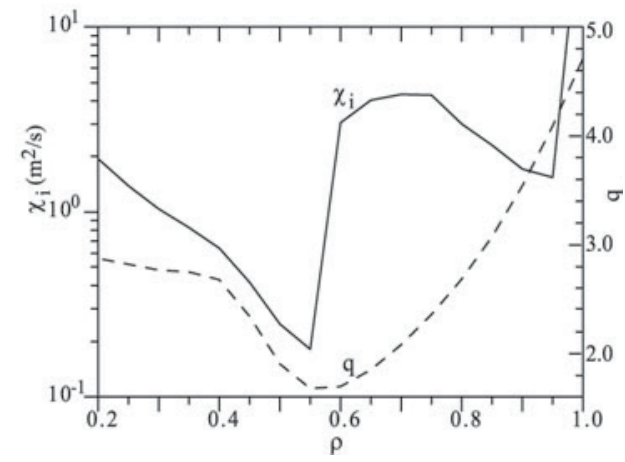

Figure 6. Profiles of $q$ and ion heat diffusivity, $\chi_{\mathrm{i}}$ (at $t=1.0 \mathrm{~s}$ ), for the case of high triangularity plasma with $\mathrm{H}$-mode edge. 
the transport barrier layers defined by $\left|\nabla T_{\mathrm{i}}\right|>\frac{1}{2} \max \left|\nabla T_{\mathrm{i}}\right|$. Comparing with the RS dicharge shown in figure 5, the RS discharge with $\mathrm{DB}$ has the following features: (1) a lower minimum $q\left(q_{\min } \approx 1.64, q_{\psi} \approx 4.7\right)$ is obtained, which is consistent with experimental evidence that the combined $\mathrm{H}$ mode edge and ITB results in broadened profiles and improved stability [9-11]; and (2) taking into account the fact that the triangularity of plasmas improves the edge pressure limit as evidenced by many experiments [2-5], the transport barrier located near the edge will enhance the plasma confinement, so that higher plasma parameters $\left(T_{\mathrm{e} 0} \sim 1.7 \mathrm{keV}, T_{\mathrm{i} 0} \sim 3.6 \mathrm{keV}\right.$, $\left.H_{98(y, 2)}=1.17-1.34, \beta_{\mathrm{N}}=1.45-1.58\right)$, compred to those in figure 5, are obtained during the stationary RS phase (figure $8)$.

Figure 8 illustrates the temporal evolution of the RS discharge with a DB. Unlike the very stable locations of both the shear reversal point and the transport barriers (here we define the position of the minimum ion heat diffusivity as the barrier location) during the stationary RS phase, the

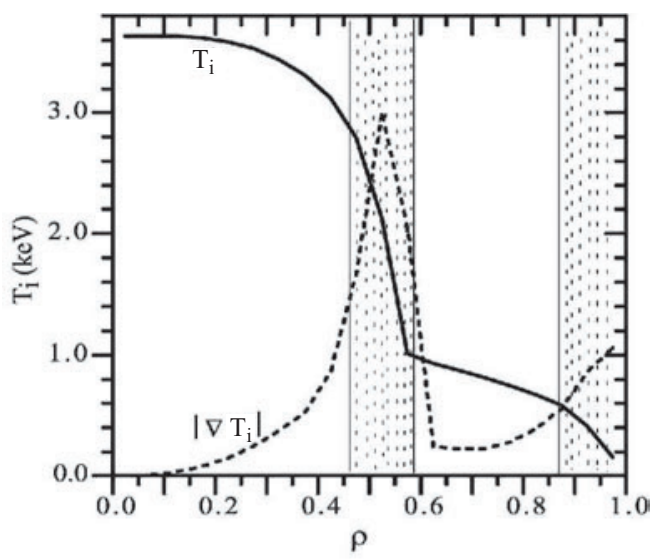

Figure 7. Profiles of the ion temperature and the gradient of the ion temperature, $\left|\nabla T_{\mathrm{i}}\right|$ (at $t=1.0 \mathrm{~s}$ ), for the case of high triangularity plasma with $\mathrm{H}$-mode edge. Shaded regions indicate the transport barrier layer.

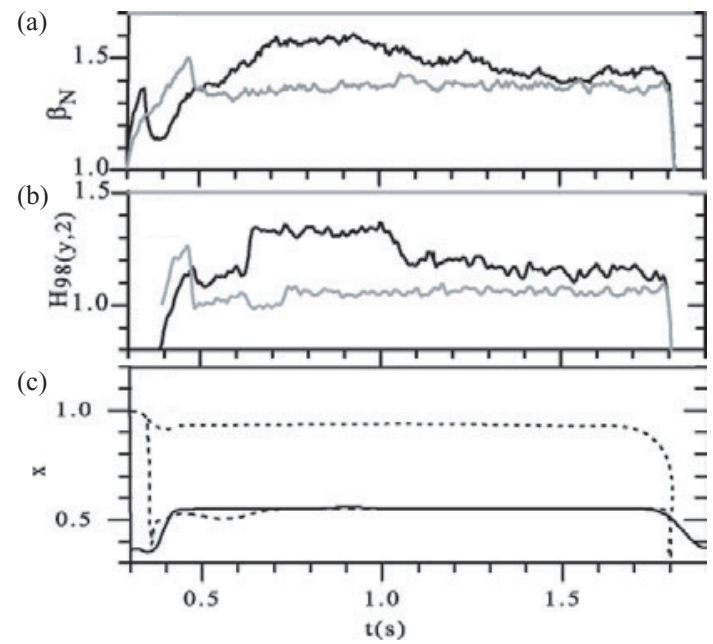

Figure 8. Time traces of an RS discharge with DB: $(a)$ normalized beta, $\beta_{\mathrm{N}} ;(b) \mathrm{H}$-factor, $H_{98(y, 2)}$; (c) locations of the DB (two dotted lines), and location of the shear reversal point (- - ). The fainter lines indicate the results of figure 5 for comparison. normalized plasma parameters $\left(H_{98(y, 2)}, \beta_{\mathrm{N}}\right)$ change with respect to time. These parameter variations are caused by the temperature profile changes that lead to variable magnitudes of the heat diffusivities. Despite the changes in plasma parameters, the RS configuration with double barriers remains stable, which shows that the RS scenario controlled by LHCD is robust.

\subsection{LH wave deposition regime in the quasi-stationary $R S$ plasma}

It turns out that, in the RS discharge modelled above, the LH wave drives stationary off-axis current with the peak of the current density located at $x>0.5$. To understand the underlying physics for the current profile control with LHCD in HL-2A, we analysed the LH wave deposition in the RS configuration. The achievement of off-axis LH power deposition in a stationary manner requires limiting the maximum allowed $n_{\|}$upshift in the central region and thus prevent wave absorption in this region. This condition can be found by calculating the $n_{\|}$boundaries of the propagation domain in phase space, where $n_{\|}$is the $\mathrm{LH}$ wave index parallel to the magnetic field.

The LH wave propagation domain is defined as the domain in phase space $(\vec{r}, \vec{k})$ where the wave phase is real. In a tokamak geometry, the appropriate canonical coordinates are $\left(r, \theta, \varphi, k_{r}, m, n\right)$, where $r$ is the minor radius, $\theta$ and $\varphi$ are the poloidal and toroidal angles, $k_{r}$ is the radial wavevector, and $m$ and $n$ are the poloidal and toroidal mode numbers [8]. By solving the wave dispersion relation $D\left(m, r, k_{r}, \omega\right)=0$ for $k_{r}$ on each flux surface for a given $n$, the region where the propagation is allowed (i.e. $k_{r}^{2} \geqslant 0$ ) is defined for the $\mathrm{LH}$ wave with frequency $\omega$. At the boundary of the propagation domain,

$$
\bar{n}_{\|}=n_{\| 0} \frac{R_{0}}{R} \frac{\hat{q}^{2} \mp \sqrt{1+\left(1+\hat{q}^{2}\right)\left(\omega_{\mathrm{pe}}^{2} / \omega^{2}\right) / \varepsilon_{\perp}}}{\hat{q}^{2}-\left[1+\left(\omega_{\mathrm{pe}}^{2} / \omega^{2}\right) / \varepsilon_{\perp}\right]},
$$

where $n_{\| 0}$ and $R_{0}$ refer to the toroidal mode number $n$ launched at the antenna $R_{0}, \hat{q}=q_{\mathrm{cyl}} / \varepsilon x \quad\left(q_{\mathrm{cyl}}\right.$ is the cylindrical safety factor, $\varepsilon=a / R)$ is a magnetic geometry factor, and $\varepsilon_{\perp}=1+\omega_{\mathrm{pe}}^{2} / \omega_{\mathrm{ce}}^{2}-\sum_{j} \omega_{\mathrm{pi}, j}^{2} / \omega^{2}\left(\omega_{\mathrm{ce}}, \omega_{\mathrm{pi}}\right.$ and $\omega_{\mathrm{pe}}$ are the electron gyro-frequency, ion and electron plasma frequencies, respectively). In deriving the above expression, the cold electrostatic approximation of the dispersion relation, $k_{\perp}^{2}=$ $k_{\|}^{2}\left(\omega_{\mathrm{pe}}^{2} / \omega^{2}\right) / \varepsilon_{\perp}$, is used, where $k_{\|}$is the wave vector component parallel to the magnetic field.

Under the conditions of the RS discharges simulated above, there is a spectral gap between the parallel LH wave phase velocity and the electron thermal velocity. To achieve off-axis LH wave power deposition in the weak damping regime, we rely on the $n_{\|}$upshift to fill the spectrum gap. Several explanations have been proposed for filling the spectrum gap, such as the broadening of the $n_{\|}$spectrum caused by anomalous Doppler instability $[26,27]$ or scattering by density fluctuations [28]. Here, the toroidal axisymmetry geometry effect, which affects the evolution of $k_{\|}$on the LH wave trajectories, is used to estimate the limit of the $n_{\|}$upshift. Using the cold electrostatic approximation, this yields

$$
n_{\|} \leqslant n_{\| 0} \frac{R_{0} / R}{1-\left(\omega_{\mathrm{pe}} / \omega\right) /\left(\hat{q} \varepsilon_{\perp}^{1 / 2}\right)} .
$$



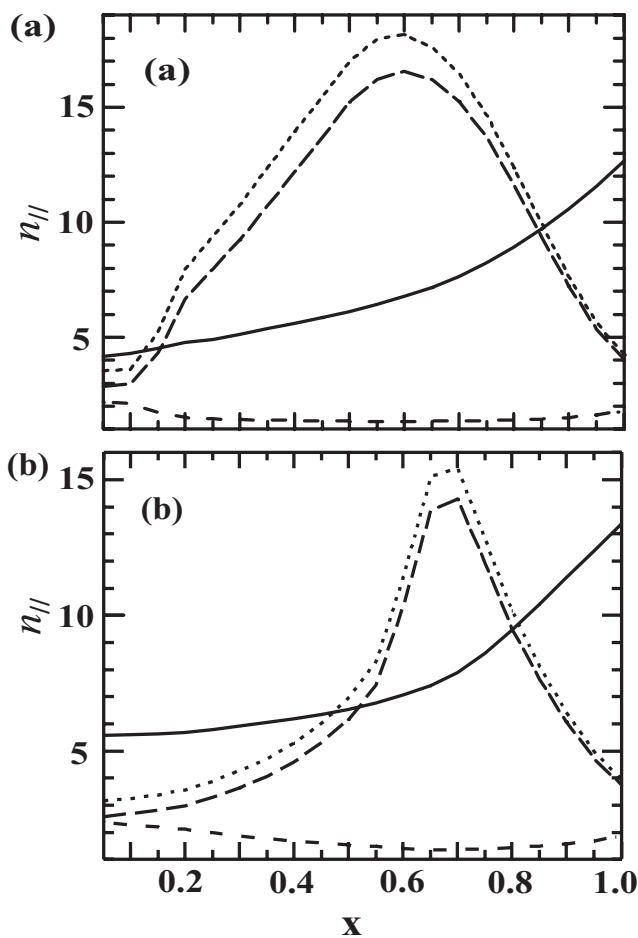

Figure 9. Regime of LH power absorption in the circular plasma with L-mode edge: ELD limit (- $-; n_{\|}$upshift boundary $(\cdots \cdots)$; boundary of propagation domain (- - - ) (at $t=1.0 \mathrm{~s})$. (a) $I_{\mathrm{p}}=300 \mathrm{kA} ;\left(\right.$ b) $I_{\mathrm{p}}=265 \mathrm{kA}$.

The LH wave absorption in a magnetized plasma depends on the electron Landau damping (ELD). If the LH wave phase velocity is higher than 3.5 times the electron thermal velocity, there are too few velocity-resonant electrons to carry the driven current density when compared with the Ohmic current density. To achieve strong Landau damping, the corresponding condition required for the current drive is

$$
n_{\|}=\frac{k_{\|} c}{\omega} \geqslant \frac{6.5}{\sqrt{T_{\mathrm{e}}[\mathrm{KeV}]}} .
$$

To show the off-axis LH wave power deposition in HL-2A, we draw the boundary of the wave propagation domain (equation (2)), the $n_{\|}$upshift boundary (equation (3)), and the ELD limit (equation (4)) in the $\left(x, n_{\|}\right)$plane (figure 9). It is shown that the LH wave absorption is bounded in the region above the ELD limit and below the boundary of the wave propagation domain. For the quasi-stationary RS operation obtained with $I_{\mathrm{p}}=265 \mathrm{kA}$, the spatial region of power deposition is limited to $0.5<x<0.8$ (figure $9(b)$ ), and it is off-axis. Nevertheless, when the plasma current increases to $I_{\mathrm{p}}=300 \mathrm{kA}$, the RS will not be maintained. Under this condition the intersection between the upper $n_{\|}$limit and the ELD limit is located at $x \sim 0.15$ (figure $9(a)$ ), and the LH power can deposit near the plasma centre, which would increase the central electron temperature, allowing the LH wave to penetrate further into the centre and generate a centrally peaked driven current. It is concluded that the variation of the LH driven current profile is due to the constraint imposed by the wave propagation domain, which can be controlled by changing the plasma parameters or the LH (a)

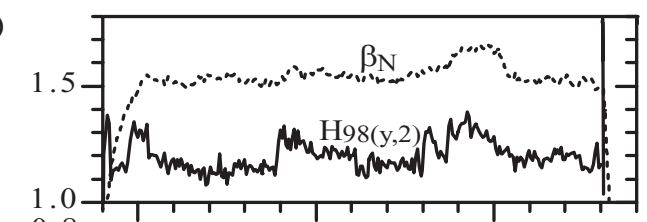

(b)

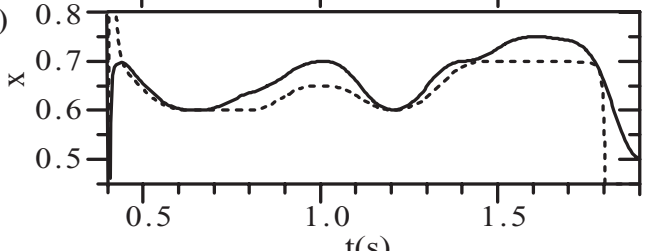

$\mathrm{t}(\mathrm{s})$

Figure 10. Temporal evolution of ( $a$ ) normalized beta, $\beta_{\mathrm{N}}$ and $\mathrm{H}$-factor, $H_{98(y, 2)}$, and (b) the location of the shear reversal point (- $)$ and the minimum ion heat diffusivity (- - - ) for a circular plasma with L-mode edge.

wave spectrum. By tuning the phasing between wave guides properly, a sustainable RS discharge with $I_{\mathrm{p}}=320 \mathrm{kA}$ can be achieved, which, although not so stationary as the discharges with $I_{\mathrm{p}}=265 \mathrm{kA}$, has higher normalized parameters, $H_{98(y, 2)}$ and $\beta_{\mathrm{N}}$ (figure 10).

\section{Conclusions}

Through shifting the plasma column outwards, magnetic geometries with significant triangularity are achieved with sufficient room left for the RF antenna. For the out-shifted shaped plasma, ripple loss of high energy ions during NBI is analysed. The results show that the ripple loss fraction of NBI power for the shaped plasma is no higher than that for the unshifted circular plasma.

The time dependent TRANSP code is used to model a realistic RS operation in HL-2A. In order to sustain the RS operation towards steady-state, off-axis current drive with a lower hybrid wave at $2.45 \mathrm{GHz}$ is used to control the current profile, and a steady-state RS discharge with an ITB is formed and sustained until the LH power is turned off. In the RS discharges with shaped plasma geometry, a DB develops. For this configuration, the combined $\mathrm{H}$-mode edge and ITB results in broadened profiles and improved stability such that a lower minimum $q\left(q_{\min } \approx 1.64\right)$ is produced and the transport barrier near the plasma edge, combined with the effect of triangularity on raising the edge pressure gradient, enhance the plasma confinement so as to achieve higher plasma parameters.

As the initial parallel phase velocity of the LH wave is sufficiently high compared to the thermal electron velocity in the HL-2A plasma centre, the wave absorption is weak and the wave makes many passes through the plasma until the initially launched wave spectrum is sufficiently broadened to be absorbed. Nevertheless, the constraint imposed by the wave propagation condition limits the maximum allowed $n_{\|}$upshift. Taking into account the Landau damping condition, an offaxis LH power deposition region is generated by choosing the plasma parameters properly, which points the way for current profile control with LHCD in establishing stationary RS operation.

In summary, the study shows that by an outward shift of the plasma column, shaped plasma cross-sections can be achieved 
with the present coil set unchanged and that ripple losses are not a problem. The study also shows that quasi-stationary magnetic shear reversal can be obtained with $\mathrm{LH}$ waves even in moderate temperature plasmas in which the waves are weakly damped. Therefore, with the aid of plasma shaping and current profile control by LHCD, stable steady-state high performance modes are produced, implying that the underlying physics of enhanced confinement in the so-called 'advanced tokamak' scenarios can be explored in the future operation of HL-2A.

\section{Acknowledgment}

This work is supported by the National Natural Science Foundation of China under Grant No 10275018.

\section{References}

[1] Gao Q.D. et al 2000 Nucl. Fusion 401897

[2] Ongena J. et al 2001 Plasma Phys. Control. Fusion 43 A11

[3] Osborne T.H. et al 2000 Plasma Phys. Control. Fusion $\mathbf{4 2}$ A175

[4] Kamada Y. et al 1997 Proc. 16th Int. Conf. on Plasma Physics and Controlled Nuclear Fusion Research 1996 (Montreal, 1996) vol 1 (Vienna: IAEA) p 247

[5] Stober J. et al 2000 Plasma Phys. Control. Fusion 42 A211
[6] Kessel C. et al 1994 Phys. Rev. Lett. 721212

[7] Turnbull A.D. et al 1995 Phys. Rev. Lett. 74718

[8] Litaudon X. et al 1996 Plasma Phys. Control. Fusion 381603

[9] Greenfield C.M. et al 2001 Phys. Rev. Lett. 864544

[10] Söldner F.X. et al 1999 Nucl. Fusion 39407

[11] Fujita T. et al 1999 Nucl. Fusion 391627

[12] Goldston R.J. et al 1981 Phys. Rev. Lett. 47647

[13] Budny R.V. et al 1995 Nucl. Fusion 351497

[14] Brambilla M. 1976 Nucl. Fusion 1647

[15] Ignat D.W., Valeo E.J. and Jardin S.C. 1994 Nucl. Fusion 34837

[16] Rebut P.H. et al 1991 Phys. Fluids B 32209

[17] Conner J.W. 1995 Plasma Phys. Control. Fusion 37 A119

[18] Manickam J. et al 1997 Proc. 16th International Conf. on Fusion Energy 1996 (Montreal, 1996) vol 1 (Vienna: IAEA) p 453

[19] Chu M.S. et al 1996 Phys. Rev. Lett. 772710

[20] Turnbull A.D. et al 1997 Proc. 16th International Conf. on Fusion Energy 1996 (Montreal, 1996) vol 2 (Vienna: IAEA) p 509

[21] Bondeson A. et al 1997 Nucl. Fusion 371419

[22] Bonoli P.T. et al 1997 Plasma Phys. Control. Fusion 39223

[23] Gao Q.D. et al 2001 Chinese Phys. Lett. 18790

[24] Ishida S. and JT-60 Team 1999 Nucl. Fusion 391211

[25] ITER Physics Basis 1999 Nucl. Fusion 392175

[26] Liu C.S. 1982 Phys. Rev. Lett. 481479

[27] Bonoli P.T. and Ott E. 1981 Phys. Rev. Lett. 46424

[28] Andrews P.L. and Perkins F.W. 1983 Phys. Fluids 262537 